\title{
Practical Determination of Spatial Resolution in Atom Probe Tomography
}

\author{
Thomas F. Kelly*, Edgar Voelk1**, and Brian P. Geiser* \\ *Imago Scientific Instruments Corporation, 5500 Nobel Drive, Madison, WI 53711-4951 USA \\ **FEI Company, 5350 NE Dawson Creek Drive, Hillsboro, OR, 97124-5793 USA
}

There are many reasons why it is important to have an established procedure that can be used by all practitioners to measure the spatial resolution of a microscopy technique. This parameter can be used to assess whether a technique can provide information at a fine enough scale to solve a question. It can be used to monitor advances in the field as the instrumentation improves. Customers of commercial instruments can use this information in assessing an instrument and in verifying its performance upon delivery. Users and suppliers can also use the information to monitor performance in the field and provide clues to what is wrong when the instrument fails to meet performance criteria.

The procedure must be easy to use, reproducible, and reveal important fundamental information at the upper limits of performance of an instrument. Vurpillot et al. [1] defined spatial resolution in atom probe tomography (APT) as the inverse of the width of the damping function that is superimposed on the reciprocal space structure of an atom probe dataset. This parameter gives information about the uncertainty in interatomic distances as determined by APT. Geiser et al. [2] introduced the spatial distribution map (SDM) as a tool to average real space information about interatomic distances in an APT dataset. The advantage of the SDM is its high signal-to-noise ratio and computational efficiency for a given dataset size. In their work, the spatial spread in the SDM about a real-space lattice point was used as a measure of spatial resolution. In fact, this latter approach is functionally equivalent to that of Vurpillot et al. [1]. Kelly et al. [3] used Fourier transforms of SDMs to describe spatial resolution in APT where the inverse of the longest statistically significant reciprocal lattice vector was called the spatial resolution but should be called the information limit in keeping with common practice in electron microscopy [4]. These three definitions all pursue the same essential information: the limits of the spatial resolution as revealed by the real space atom location uncertainty/reciprocal space damping function. Also, there are two physically different mechanisms at play in resolving information in the longitudinal and lateral directions of APT, respectively, and measurements for both are needed.

Spatial resolution is a local measurement. That is, it should express the ultimate performance of the instrument and not be influenced by other sources of imperfection such as imperfect reconstruction procedures or trajectory aberrations near a low-index zone axis. For these reasons, we propose that a standard measurement of spatial resolution in APT utilize an SDM from the best section of the idealspecimen image. It should utilize the smallest possible volume for the SDM. However, as the analyzed volume of the SDM decreases, the signal diminishes. We further propose that the smallest equiaxed cylindrical volume that will still give the $<100>$ peak in a W SDM with signal-to-noise ratio $(\mathrm{S} / \mathrm{N})$ better than the limit defined by Curie [5], i.e., $\mathrm{S} / \mathrm{N}>3$. The spatial resolution is then defined as the full width at half maximum (FWHM) of the $<100>$ peak. Since the four nearestneighbor atom positions are all identical, the average of these peaks should be used.

The base temperature of the specimen will have a strong influence on the spatial resolution achieved and must therefore always be specified. We recommend that the lowest temperature be used and $30 \mathrm{~K}$ is generally achieved. Because field (or voltage) pulsing, as opposed to thermal pulsing, yields the 
best spatial resolution, it is the recommended standard pulsing mode. Furthermore, these measurements would ideally assess an instrument's performance and not depend on the specimen: the specimen should act like an impulse function. This ideal specimen should be readily available, evaporate well and yield many crystal planes resolved to high order in real space. Tungsten is as close to this ideal sample as we know. It also has a strong texture along $<110>$ when in wire form that means most specimens will have the same $<110>$ crystal orientation. As a monatomic material, pure tungsten will not have heterophase trajectory aberrations.

We have taken a data set that meets the above criteria and made preliminary assessments of these concepts. Fig. 1 shows SDMs and intensity profiles through the lattice points. The diameter of the cylindrical volume was reduced successively and the impact on the visibility of the peaks in the SDM was evaluated. The results in Fig. 1e,f were almost at the limit of peak visibility. The FWHM of these peaks was determined to be $0.15 \mathrm{~nm}$ in this case.

[1] F. Vurpillot et al., J. Microsc., vol. 203, pt. 3 (2001) pp. 295-302.

[2] B. Geiser et al., Microsc. Microanal., vol. 13, no. 6 (2007) pp. 437-447.

[3] T.F. Kelly et al., Microsc. Microanal., 13(Suppl 2) pp. 1604-1605 CD.

[4] S. Van Aert et al., in "Science of Microscopy," P.W. Hawkes and J.C.H. Spence, eds., Springer (2007) pp. 1228-1265.

[5] L. A. Currie, Anal. Chem., vol. 40 (1968) p. 586.
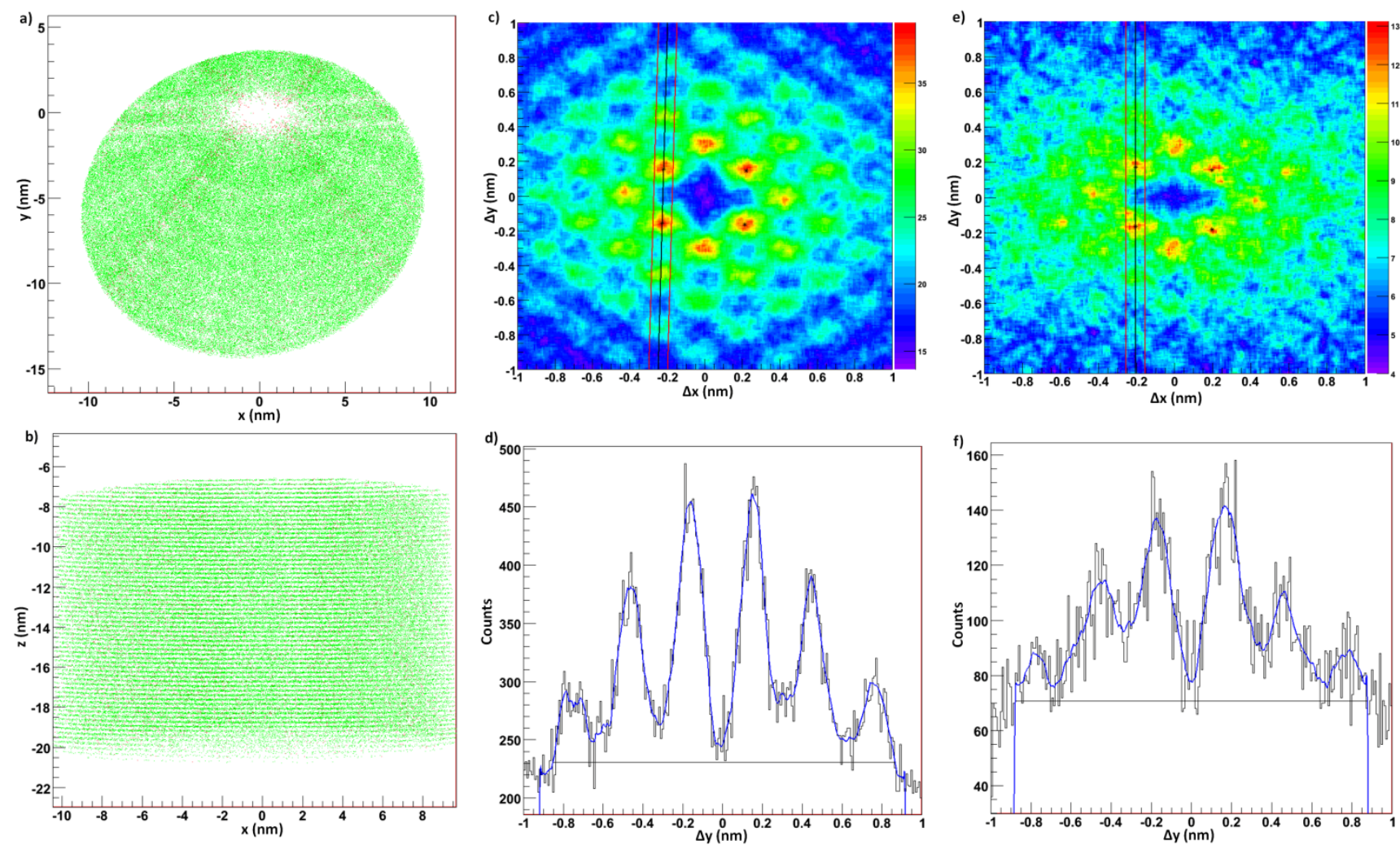

Fig. 1 a) Longitudinal view and b) lateral view of the APT image with 151,198 atoms. c) Longitudinal view of basal plane of $\operatorname{SDM}(\Delta(\Delta \mathrm{z})=0.01 \mathrm{~nm})$ of a) and d) intensity line scan through $<100>$ peaks as depicted in c). e) Repeat of c) and d) for APT image of $1 / 2$ the diameter centered on the same point as a) with 42,898 atoms. 